\title{
Encoding Energy-Density as Geometry
}

\author{
Edwin Eugene Klingman (1) \\ Cybernetic Micro Systems, Inc., San Gregorio, CA, USA \\ Email:klingman@geneman.com
}

How to cite this paper: Klingman, E.E. (2021) Encoding Energy-Density as Geometry. Journal of Modern Physics, 12, 1190-1209. https://doi.org/10.4236/jmp.2021.129073

Received: June 1, 2021

Accepted: July 5, 2021

Published: July 8,2021

Copyright $\odot 2021$ by author(s) and Scientific Research Publishing Inc. This work is licensed under the Creative Commons Attribution International License (CC BY 4.0).

http://creativecommons.org/licenses/by/4.0/

\begin{abstract}
Physicists possess an intuitive awareness of Euclidian space and time and Galilean transformation, and are then challenged with Minkowski space-time and Einstein's curved space-time. Relativistic experiments support the "time-dilation" interpretation and others support "curved space-time" interpretation. In this, and related work, we investigate the key issues in terms of the intuitive space-time frame. In particular, we provide alternative approaches to explain "time dilation" and to explain the energy density for gravity systems. We approach the latter problem from an information perspective.
\end{abstract}

\section{Keywords}

Curved Space-Time, Gravitational Energy, Stress-Energy Tensor, Encoding Information in Geometry, Time Dilation, Equivalence Principle, Minkowski Relation, Schwarzschild Metric, Linearized General Relativity, Cosmology

\section{Introduction}

Every general relativity text contains some discussion of the fact that the energy of gravitating systems cannot be formulated in curved space-time. A recent Centennial paper [1] by Chen, et al. begins by stating:

"How to give a meaningful description of energy-momentum for gravitating systems... has been an outstanding fundamental issue since Einstein began his search for gravity theory."

The problem of how best to describe the energy-momentum and angular momentum in gravitating system suffers from the fact that

"It is known that these quantities cannot be given a local density."

A century-long failure to solve the fundamental problem is indicative of confusion; the goal of this work is to provide necessary clarification of the issue. The plan for this paper is as follows:

Section 2 introduces coordinate systems and their meaning, in the context of 
invariance. We review the development of energy-time physics in absolute space and time, based on treating the Minkowski relation as an invariance, not as space-time.

Section 3 provides context for the geometric algebra approach applied to the problem of "clock slowdown" in moving systems. Two interpretations of the same geometric algebra construct yield two or more conceptions of physics based on corresponding ontological assumptions.

Section 4 reviews issues of coordinates in theories that formulate gravity as curved space-time. These issues are at the root of the problem of gravitational energy representation in curved space.

Section 5 presents the essence of the "never-solved" problem of general relativity; the fact that a century of effort has failed to formulate a meaningful description of energy-momentum for gravitating systems.

Section 6 introduces Einstein's equations and focuses on their inherent insolvability except in One-body solutions for essentially trivial cases.

Section 7 begins the central effort in this work: the encoding of gravitational energy density in geometry. It formulates the density for the primordial field and one body of mass $M$ located in the field. The procedure for encoding the energy density in the geometry is prescribed.

Section 8 analyzes the encoding scheme of Section 7 and formulates the appropriate equations connecting flat-space coordinates to curved-space coordinates. The equations are shown to be equivalent to the Schwarzschild metric solution to Einstein's equation.

Section 9 derives the time metric $\left(g_{00}\right)$ associated with curved 3-space and shows it to match the Schwarzschild metric.

Section 10 discusses the time metric as a function of position in the local field. Section 11 discusses the conclusions we draw from this work.

\section{Intro to Coordinate Systems and Their Meaning}

One of the fundamental rules of physics is that coordinate systems have no effect on physical reality. When this is violated, physics becomes confusing. We begin by noting that Euclidean space is Pythagorean in that distances in space and time are unlimited.

$$
\mathrm{d} s^{2}=c^{2} \mathrm{~d} t^{2}+\mathrm{d} x^{2}+\mathrm{d} y^{2}+\mathrm{d} z^{2} \rightarrow \infty
$$

(Pythagorean distance in Euclidean space and time)

This implies that Euclidian four-space can be mapped onto any and all events in the universe. That allows us to label every event and to relate any event to any other. These relations constitute our physics or models of reality. But what are the relations? They are intended to capture objective reality in some sense. Nozick, in "Invariance: the structure of the objective world" [2] observes that an objective fact is accessible from different angles, i.e., "an objective fact is invariant under various transformations."

Consider the Galilean transformation describing a photon's position in 4-space 
with time

$$
\vec{x}=\vec{v} t
$$

where velocity $\vec{v}$ can point in any direction and $| \pm \vec{v}|=c$. Thus $x=-c t$ represents the photon moving in the negative direction. Rearranging and taking a difference we have

$$
\mathrm{d}(x-c t)=0 \text { or } \mathrm{d}(x+c t)=0
$$

Following Hestenes, [3] we define geometric algebra multi-vector $X=c t+\vec{x}$ and its complement $\tilde{X}=c t-\vec{x}$. It is simple to show that

$$
\mathrm{d} X \mathrm{~d} \tilde{X}=c^{2} \mathrm{~d} t^{2}-\mathrm{d} \vec{x}^{2}=0
$$

This is an invariance that holds for all distances $\mathrm{d} \vec{x}$ and corresponding duration $\mathrm{d} t$. It is, of course independent of coordinate systems. If we denote the value $\mathrm{d} X \mathrm{~d} \tilde{X}$ by $\mathrm{d} s^{2}$ we obtain the invariance relation for the photon equation of motion (where we use $\mathrm{d} \vec{x}^{2}=\mathrm{d} x^{2}+\mathrm{d} y^{2}+\mathrm{d} z^{2}$ ):

$$
\mathrm{d} s^{2}=c^{2} \mathrm{~d} t^{2}-\mathrm{d} x^{2}-\mathrm{d} y^{2}-\mathrm{d} z^{2}=0
$$

From the above discussion we interpret this as an invariance relation in Euclidian 4-space and we call it the Minkowski invariance. But Minkowski did not conceive of this as an invariance relation; he actually believed it was a description of "space-time", and famously stated: "space and time by itself are doomed to fade away... only a kind of union of the two will preserve an independent reality." In fact, applying the Lorentz transformation to this imagined space time he showed that space can be rotated into time and time rotated into space. Little wonder physicists who grew up with an intuitive grasp of Euclidian space and time found it difficult or impossible to grasp Minkowski space-time.

In "Physics of clocks in absolute space and time" [4] I choose Equation (5) as the fundamental photon-based invariance and use Hestenes' multi-vector to derive the classical Hamiltonian

$$
H=E=\sqrt{m_{0}^{2} c^{4}+c^{2} p^{2}} .
$$

This is then used to show that if two clocks are built to tell identical time when side-by-side, and one clock is accelerated to velocity $\vec{v}$, the time interval read on the moving clock, $\mathrm{d} \tau$, will run more slowly than the time duration $\mathrm{d} t$ measured on the clock at rest according to

$$
\frac{\mathrm{d} \tau}{\mathrm{d} t}=\gamma=\left(\frac{1}{\sqrt{1-\left(v^{2} / c^{2}\right)}}\right)
$$

The physics used to derive this "time dilation" is based on Galilean transformations in absolute time and space and the "relativistic" inertia

$$
m=\gamma m_{0}
$$

where $m_{0}$ is the rest mass. This result agrees with Lucas and Hodgson [5]:

"If we... retain Newtonian dynamics, and the Newtonian definition of velocity and acceleration, then we... still obtain relativistically correct results if we... al- 
low the mass to depend on the velocity."

In special relativity rest mass is defined in all inertial reference frames in relative motion, and the Lorentz transformation is applied to "space" and "time", where space-time is imagined as defined by Minkowski. In the referenced energy-time derivation, the most solid experimental fact of special relativity, time dilation, is reproduced exactly in absolute space and time without paradoxes that mar special relativity. Energy-time theory does not yield length contraction nor Einstein's law of velocity addition; Rindler [6] says length contraction will probably never be tested; Einstein's law of velocity addition is known to be violated in LHC-type accelerators [7].

The physics of "clock slowing" in absolute time (universal simultaneity) and absolute space (with preferred frame defined by local gravity) follows from increased inertia of the clock mechanism and consequent decrease in acceleration of the restoring force common to all harmonic oscillators. Energy-time physics is compatible with all relativistic experiments [8], yet is based on Galilean transformation in intuitive time and space, in which the Minkowski relation is an invariance relation.

\section{Remarks on Geometric Algebra Approach}

Hestenes, as noted, formulates a geometric algebra multi-vector consisting of the scalar $c t$ and vector $\vec{x}: X=c t+\vec{x}$. With its complement $\tilde{X}=c t-\vec{x}$ he derives the Minkowski invariance. Writing circa 1965, he was focused on showing that his mathematics is compatible with special relativity; he was not interested in reinterpreting relativity. He therefore viewed Minkowski as "space-time" and the Lorentz transformation as operating on space-time.

We, on the other hand, began in search of an invariant upon which to base physics in Euclidean space and time. Hestenes' formulation leads immediately to such invariance. After almost a century of proof of "time dilation" we know that clocks in motion run slower than clocks at rest. Therefore we extend the invariance to frames in relative motion by assigning each frame clock reading $\mathrm{d} t=$ duration in rest frame, $\mathrm{d} \tau=$ duration for moving clock. We find that invariance in relative frames leads to $\mathrm{d} \tau / \mathrm{d} t=\left(1-v^{2} / c^{2}\right)^{-1 / 2}$. Hestenes' multi-vector formulation leads to the classical Hamiltonian in intuitive space and time, and is compatible with $m=\gamma m_{0}$.

Recognizing that all clocks count cycles and that cycles derive from simple harmonic oscillator mechanisms, it is easy to show that increased inertia of the material oscillator leads to resistance to the acceleration of the oscillator restoring force, and hence clocks in motion run slower than clocks at rest. Moreover they do so by exactly a factor of $\gamma$. In other words Hestenes' multi-vector formulation of time and space leads to special relativity if viewed as Minkowski spacetime operated on by Lorentz transformation, but leads to classical physics if treated as Minkowski invariance.

$$
X=c t+\vec{x} \quad \text { Minkowski space }+ \text { Lorentz transformation } \Rightarrow 4 \text { D relativity }
$$


$X=c t+\vec{x}$ Minkowski invariance + Euclidean space + Galilean $\Rightarrow(3+$ 1)D reality

Observe that the mathematical construct representing time and space is the same; the ontological assumptions determine what one does with it.

Amazingly, most treatments of special relativity openly discuss built-in paradoxes, where paradox is synonymous with logical contradiction, yet special relativity is held as sacred and our intuition is dismissed as misleading. The paradox-free theory summarized above is compatible with our intuition and compatible with all physics experiments; therein the Minkowski relation is not a description of space-time, but a photon-based invariance relation at the heart of physics.

\section{Coordinates and Gravity as Curved Space-Time}

In "The Schwarzschild metric. it's the coordinates, stupid", Will et al. [9] observe that

"Every general relativity text emphasizes that coordinates have no physical meaning... Every student of general relativity is taught that coordinates are irrelevant to physics."

"The principle of general covariance, upon which general relativity is built, implies that coordinates are simply labels of space-time events that can be assigned completely arbitrarily. The only quantities that have physical meaning - the measurables-are those that are invariant under coordinate transformations." It is such invariance that we used to derive the physics of absolute space and time in reference 4 . That approach led to physics of energy-time rather than the space-time focus of relativity. The success of this theory in matching the experimental data of the last century suggests that we apply the focus on energy to the general relativistic treatment of gravity.

Despite the catechism that coordinates can have no effect on physics, the equivalence principle upon which general relativity is typically founded yields a built-in contradiction: by transforming local coordinates the gravitational field can be banished. Weinberg [10] formulates the equivalence principle as

"At every space-time point in an arbitrary gravitational field it is possible to choose a 'locally inertial coordinate system' such that [locally] the laws of nature take the same form as in unaccelerated Cartesian coordinate systems in the absence of gravitation."

In other words, despite grand statements to the effect that coordinate systems have no effect on physics, the proper choice of a locally inertial coordinate system effectively makes gravity vanish! Poisson and Will [11] state:

"All local aspects of gravity can be turned off by doing physics in a freely moving (coordinate) frame of reference... Gravity is not present in these (coordinate) frames."

In other words, one can always mathematically do away with the local gravitational field and hence any associated energy. A consequence of this is ambiguous 
treatment of gravitational energy in general relativity, a problem that has never been solved.

\section{The Essence of the "Never-Solved" Problem}

Chen et al. observe in "Gravitational energy for GR...", that in all of Einstein's published papers, "most include a significant consideration of the topics of gravitational energy."

Most physicists are aware of Emmy Noether's seminal work on symmetry and conservation. What most probably do not realize is that the reason Noether began investigations was "to clarify the issue of gravitational energy." In fact, in 1918 she proved that "there is no covariant total energy-momentum density tensor for gravitating systems." MTW [12] discuss this feature as "a consequence of the equivalence principle..."

A century of effort in this direction has produced many results; however it has not solved the problem. One might say physics has given up on the problem; Penrose in 1982 invented the idea that "energy-momentum is quasi-local: i.e., it is associated with a closed 2-surface...". Finding an appropriate quasi-local notion of energy-momentum has been surprisingly difficult. In fact,

"the state-of-the-art is typically postmodern: although there are several promising and useful suggestions, we not only have no ultimate, generally accepted expression for the energy-momentum and especially for the angular momentum, but there is not even a consensus in the relativity community on general questions... or on the list of the criteria of reasonableness of such expressions."

The remainder of this paper will examine the nature of the problem that has frustrated general relativists for over a century.

\section{Introduction to Einstein's Equations}

A non-vanishing energy-density necessarily produces gravity (i.e., the curvature of space-time). It is questionable whether most physicists even have an intuitive conception of curved spacetime. Can a re-interpretation of general relativity clarify "curved space-time" and bring it into congruity with our intuitive ideas of space and time? Recall that Einstein's vision of gravity is pure geometry. His basic equation

$$
G_{\mu v}=\kappa T_{\mu v}^{(m)}
$$

specifies that stress-energy tensor $T_{\mu \nu}^{(m)}$ defines the distribution of mass-energy while $G_{\mu \nu}$ defines the geometry of the "curved space-time" induced by the existence of the material stress-energy $T_{\mu \nu}^{(m)}$. The problem is to derive the curved space geometry from $T_{\mu \nu}^{(m)}$. Lorentz, Levi-Civita and Klein argued that the Einstein curvature tensor $G_{\mu \nu}$ was the only proper gravitational energy-momentum density; hence one should regard the Einstein equation in the form

$$
-\kappa^{-1} G_{\mu v}+T_{\mu \nu}^{(m)}=0
$$

as describing the vanishing sum of gravitation and material energy-momentum. 
However, this is generally self-contradictory if the solution is in curved space-time and curvature is known only after the problem is solved; how could we possibly express the source distribution in the not yet solved for curvature geometry? We cannot solve for curvature without knowing the source; and cannot express source distribution $T_{\mu v}^{(m)}$ responsible for curvature in the curved space that is the object of solution. How is this problem handled in relativity? Chen et al. observe:

"Specifically, in the case of GR, knowing the curvature gives, via the Einstein tensor, the symmetric Hilbert energy-momentum density."

This does not solve the problem. It states that, if the problem could be solved, and we knew the solution ("knowing the curvature") then we could represent the energy-momentum density. This is not entirely un-akin to the observation that, "if we had some ham, we could have ham and eggs, if we had some eggs." It definitely does not solve the never-solved problem of gravitational energy density that induces curvature of space-time.

Feynman [13]: gravity theory suffers because

"... one side of the equation is... geometric, and the other side is not [geometric]... even for very simple problems, we have no idea how to go about writing down a proper $T^{\mu \nu}$."

Little wonder physicists generally have no intuitive conception of curved space-time. There are two possible solutions; a trivial and a non-trivial solution. The trivial solution is $G_{\mu \nu}=0$, where the stress-energy tensor is everywhere zero in all coordinate frames. This generally implies "flat space" but Vishwakarma has analyzed curvature in [14] in view of the fact that a proper energy-stress tensor of the gravitational field does not exist. He discusses the Kasner solution, which I have treated in [15], "A Primordial Space-Time Metric". As noted in his title, this trivial solution involves "a new paradigm in $G R$ ".

There is also a nontrivial way to avoid the paradox of solving an equation expressed in two nontrivially different coordinate systems. I observe that the only non-trivial way to avoid this paradox is to place the center of mass of a spherically symmetric body at the only point common to both flat space and curved space, the origin, and appeal to Birkhoff's Shell theorem. This inherently limits Einstein's general relativity to One-body solutions of the $\mathrm{N}$-body problem.

Heaviside [16] extended Newtonian physics to include field energy density in flat space, and this is recognized as iteratively equivalent to Einstein's non-linear field equation. Feynman noted:

"It is one of the peculiar aspects of the theory of gravitation, that it has both a field interpretation and a geometric interpretation..."

It is not generally clear how a density-based field description is related to metric curvature solutions; our immediate goal is to clarify this.

\section{Encoding Density in Geometry}

We ask how one might understand the transition from physical-field-based 
physics in flat space to curved-space-time-geometry-based physics. Despite the common perception that Minkowski space-time views space as empty, Einstein

[17] came to realize that

"There is no such thing as empty space, i.e., a space without a field. Space-time does not claim existence on its own, but only as a structural quality of the field."

Further, physical fields are real and have energy. Ohanian and Ruffini [18]

"The gravitational field may be regarded as the material medium sought by Newton; the field is material because it possesses an energy density."

In other words, gravity is viewed not as curved space-time, but as a field with energy density. Yet special relativity is based on pretending that gravity does not exist at all; and general relativity on pretending that gravitational energy does not exist "locally"-it can always be transformed away. This despite universal agreement that coordinate systems, curved or flat, can have no effect on physical reality. The logical contradiction that a change in coordinate system can make a physically real field vanish is hidden in the Equivalence Principle used to derive our premier theory of gravity. The claim of non-local energy density yields "gravity as space-time curvature".

Despite recent focus [19] on "information" as a real physical entity, we examine information in its original coding perspective; we observe that there is absolutely no energy density information encoded in flat space coordinates. One can change the coordinate system with absolutely no effect on the field energy density; one can change the energy distribution with absolutely no effect on the coordinate system. All of the information about the physical field is contained in the energy density distribution; none in the flat space coordinates!

In Table 1, flat space information is in the energy density; no information (other than scale) is associated with units of the coordinate system. In curved space, normalized energy represents no information; the relevant information is encoded in the geometry specified by the metric.

What happens to the physics when we remove density information by claiming zero local energy density? It must be replaced somehow! If the only physical info we have is energy density and coordinate info, then when removing the density info we must replace it with coordinate info, and this is done by replacing constant length flat space coordinates with variable length (metric) curved space coordinates. No information has been lost; the real physical information encoded by the density distribution over flat space has been replaced by abstract information in which physical energy density is encoded as "geometry". According to Poisson and Will:

Table 1. Information matrix.

\begin{tabular}{ccc}
\hline & Flat & Curved \\
\hline energy density & 1 & 0 \\
metric length & 0 & 1 \\
\hline
\end{tabular}


"the metric... achieves two purposes: it encodes geometrical information about coordinate system, and it encodes physical information about the gravitational field."

I would say that the metric encodes physical information about the gravitational field as geometrical information in a coordinate system. This clearly recognizes that the physical field energy density is ontologically real, and is mathematically equivalent to the description of the real physical density encoded in the abstract formulation of curved space time!

One best distinguishes physical density-based reality from curved space-timebased reality via inertia. In Energy-time theory the special relativity $\gamma$-factor applies to inertial mass. In curved spacetime real inertial force is replaced by the abstraction of geodesics. This is at the root of Feynman's, Weinberg's, Padmanabhan's, and others insistence that

"Curved space-time is not a necessary conception of gravity."

Consider the primordial gravitational field treated in "The Primordial Principle of Self-interaction" [20]. The Self-interaction equation $\vec{\nabla} \vec{\psi}=\vec{\psi} \vec{\psi}$ is used to derive the Heaviside equations known to be equivalent under iterations to Einstein's field equations. The geometrical algebra formulation is the multi-vector $\vec{\psi}=\vec{G}+i \vec{C}$ where $\vec{G}$ is the gravitational field vector and $\vec{C}$ is the gravitomagnetic bivector. The duality operator $i$ converts $\vec{C}$ to $\vec{C}$. In the absence of circulation $\vec{C}$ the solution to $\vec{\nabla} \vec{\psi}=\vec{\psi} \vec{\psi} \Rightarrow \vec{\nabla} \vec{G}=\vec{G} \vec{G}$ is $\vec{G}=1 / \vec{r}\left(\equiv \vec{r} / r^{2}\right)$. Hence $\vec{G} \vec{G}=1 / r^{2}$ is the energy density of the primordial field. As shown in Figure 1, the mass of the field inside a sphere of radius $r$ is (modulo $4 \pi / 3$ )

$$
m \cong r^{3}(\rho)=r^{3}(\vec{G} \vec{G})=r^{3}\left(\frac{1}{r^{2}}\right)=r \Rightarrow \frac{m}{r} \cong 1
$$

Thus potential $\phi=-(m / r \cong 1)$ where Newton's gravitational constant $g=1$ and speed of light $c=1$. Let us assume that this energy is distributed with unit density. Consider a volume defined by $\mathrm{d} x \mathrm{~d} y \mathrm{~d} z$ where $\mathrm{d} x=1, \mathrm{~d} y=1, \mathrm{~d} z=1$ as shown in Figure 2. Ignoring the sign of $\phi$ we define unit energy density of the primordial gravitational field with no material objects in the field.

Recall that the difference in potential energy of two points is the work done in moving mass $m$ between two points.

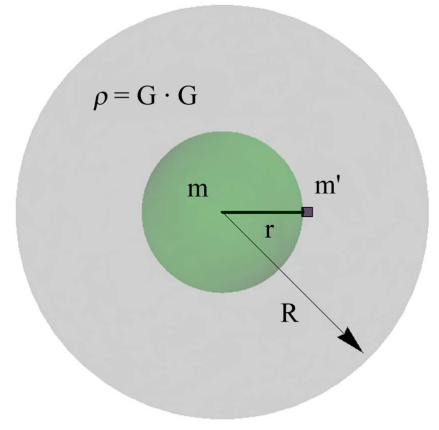

Figure 1. Mass $m$ at $\vec{r}$. 


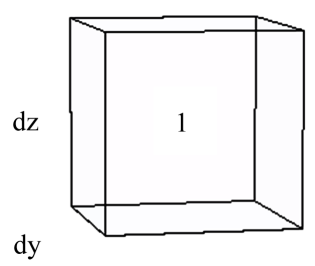

$\mathrm{dx}$

Figure 2. The energy density of the primordial gravitational field is chosen to be unity in a unit differential volume.

$$
U(r)=W_{\infty r}=\int_{\infty}^{r} \vec{F} \cdot \mathrm{d} \vec{r}=\int \frac{g M m}{r^{2}} \mathrm{~d} r=g M m\left[-\frac{1}{r}\right]_{\infty}^{r}=-\frac{g M m}{r}=m \phi
$$

The "gravitational energy per unit mass", $\phi$ describes the potential energy at the point in question. This is the interaction energy $m \phi$ that would exist if $m$ units of mass were to be introduced at that point. In the literature, this "test mass" $m$ is presumed small enough that its own gravitational potential need not be taken into account.

Next we add a mass $M$ to space. As noted, the only point common to both flat space and curved space is the origin $(0,0,0)$, assumed coincident. Although the massive body $M$ does not exist at a point we assume it is symmetrical and thus we apply Birkhoff's Shell theorem to reduce $M$ to an equivalent point mass. At distance $r$ from the origin the gravitational energy (per unit mass) is $\phi=-M / r$. Figure 3 represents the energy of the primordial field and mass $M$.

The total gravitational energy in a given region is the sum of the primordial gravitational field plus the potential due to mass $M$ located at the origin

$$
\frac{m}{r}+\frac{M}{r} \Rightarrow 1+\phi
$$

We've chosen the unit cube $\mathrm{d} x \mathrm{~d} y \mathrm{~d} z$ where $\mathrm{d} x=1, \mathrm{~d} y=1, \mathrm{~d} z=1$ as the region containing energy density $1+\phi$. We have suppressed the negative sign of the potential(s) to simplify the treatment, but we will find [in Equation (16)] that the term of interest is always positive, whether we use $(1+\phi)$ or $-(1+\phi)$. Our goal is to normalize this density $1+\phi \rightarrow 1$ by choosing new variable coordinates such that $\mathrm{d} x^{\prime} \neq 1, \mathrm{~d} y^{\prime} \neq 1, \mathrm{~d} z^{\prime} \neq 1$. Based on the equivalence principle one might assume that $1+\phi \rightarrow 0$ since "local density" cannot be defined. This is not the same as saying that local energy density of the field disappears; it merely ceases to provide physical information. We normalize the energy in every relevant volume, such that $\mathrm{d} x^{\prime} \mathrm{d} y^{\prime} \mathrm{d} z^{\prime}$ always contains unit energy density as shown on the right side of Figure 4.

In this way the information has been transformed from the energy density of the field $\phi$, in constant (informationless) coordinates of flat space, to the information of the (variable) coordinates containing a constant (informationless) energy density in curved space-time. This is consistent with the fundamental requirement that coordinate systems cannot affect physical reality. They bring no information to reality; they only label physical reality such that we all agree 


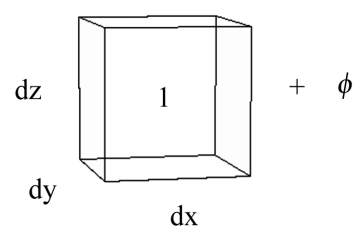

Figure 3. Unit primordial field plus local $M$ field.

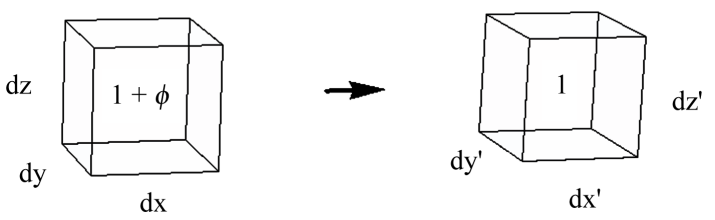

Figure 4. The primordial gravitational field alone has $\phi_{0}=m / r=r / r=1$ shown in Figure 2 in flat space, $\mathrm{d} x, \mathrm{~d} y, \mathrm{~d} z=1$. For mass $M$ at the origin, we add potential energy $\phi=M / r$. The goal is to transform to a unit density (no density information) in curved space-time as shown in the cube at right, $\mathrm{d} x^{\prime}, \mathrm{d} y^{\prime}, \mathrm{d} z^{\prime} \neq 1$.

upon the points under discussion. Of course, depending upon the physical configuration involved, one coordinate system, say spherical, may be more convenient (easier calculations) than another (say cylindrical) but neither coordinate system contains any more information than the other.

One might ask why the value 1 is used for the apparent energy density in curved space instead of the value zero that seems to be implied by the equivalence principle. We do not require that the energy density vanish, only that information about the energy density must vanish, in accordance with Einstein's statement that "gravitational energy is not localizable". Thus we are not working to get rid of the physical energy density; we are working to get rid of the energy density information. This is accomplished by normalizing the energy density such that every local region in curved space contains exactly one unit of energy density in a region bounded by $\mathrm{d} x^{\prime}, \mathrm{d} y^{\prime}, \mathrm{d} z^{\prime} \neq 1$, as shown at right in Figure 4. By defining $\mathrm{d} x_{i}^{\prime}$ to accomplish this goal we transfer the physical density information in flat space to the curvature (metric) information of curved space. In flat space every differential has unit length and there is no information contained in the normalized unit of the coordinate system. In curved space, the metric intervals are defined to normalize the energy density of the field. This removes the information from the energy density and transfers it to the curved space metric.

\section{Analyzing the Encoding Scheme}

We ensure that "gravitational energy is not localizable" by requiring that, in curved space-time, every local region contains a unit of energy indistinguishable from any other local region, whereas the local metric at any point is distinguishable from the metric at any neighboring point. This is captured by the expression of the generalized Pythagorean for curved space-time:

$$
\mathrm{d} s^{2}=g_{\mu \nu} \mathrm{d} x^{\mu} \mathrm{d} x^{\nu} \text {. }
$$


The goal, of course, is to calculate the metric $g_{\mu v}$ necessary to transform the energy density information contained in $\phi$ into the local coordinate information contained in $g_{\mu \nu}$. Whereas $\mathrm{d} x \mathrm{~d} y \mathrm{~d} z$ coordinate differentials are independent of $\phi$, the transformed coordinates are totally dependent on $\phi$, i.e.,

$$
\mathrm{d} x^{\prime} \mathrm{d} y^{\prime} \mathrm{d} z^{\prime} \equiv \mathrm{d} x^{\prime}(\phi) \mathrm{d} y^{\prime}(\phi) \mathrm{d} z^{\prime}(\phi) .
$$

For the most general solution we force each metric-based component to satisfy the relations

$$
\begin{aligned}
& (1+\phi) \mathrm{d} x_{1}^{\prime}(\phi)=\mathrm{d} x_{1} \\
& (1+\phi) \mathrm{d} x_{2}^{\prime}(\phi)=\mathrm{d} x_{2} \\
& (1+\phi) \mathrm{d} x_{3}^{\prime}(\phi)=\mathrm{d} x_{3}
\end{aligned}
$$

In this case the key coordinate variable relation becomes

$$
\mathrm{d} x_{i}^{\prime}(\phi)=\frac{\mathrm{d} x_{i}}{1+\phi}
$$

The consequent volume element becomes

$$
\mathrm{d} x_{1}^{\prime}(\phi) \mathrm{d} x_{2}^{\prime}(\phi) \mathrm{d} x_{3}^{\prime}(\phi) \equiv \frac{\mathrm{d} x_{1}}{1+\phi} \frac{\mathrm{d} x_{2}}{1+\phi} \frac{\mathrm{d} x_{3}}{1+\phi} .
$$

And for any two of these dimensions we obtain the (always positive) product:

$$
\mathrm{d} x_{i}^{\prime}(\phi) \mathrm{d} x_{j}^{\prime}(\phi) \equiv \frac{\mathrm{d} x_{i}}{1+\phi} \frac{\mathrm{d} x_{j}}{1+\phi} .
$$

The above analysis, restricted to 3-space, is effectively a "time-slice" of Euclidian reality, $\mathrm{d} t \equiv 0$; we use the convention $i, j \in\{1,2,3\}, \mu, v \in\{0,1,2,3\}$. The subtlety of 4-dimensional space-time certainly contributes to the difficulty of reconciling Einstein's theory with our intuitive ideas of time and space. As we've only considered 3-space energy density we begin with the Pythagorean relation $\mathrm{d} s^{2}=g_{\mu \nu} \mathrm{d} x^{\mu} \mathrm{d} x^{\nu}$. From Equations (11) and (16) we see that

$$
g_{i i}=\frac{1}{(1+\phi)^{2}}=(1+\phi)^{-2} \text {. }
$$

In order to make sense of this we quote John Wheeler's remarks about a white dwarf star: "It is small, but not terribly small; dense, but not terribly dense. Space-time is 'flat' within it..." The term "flat" means $\phi$ is small, approaching zero, therefore we use the binomial expansion

$$
(1+\phi)^{-2} \cong(1-2 \phi) \text {. }
$$

Yilmaz, [21] in his "Field theory of Gravity" derives a metric proportional to $\mathrm{e}^{-2 \phi}$; for consistency with consensus GR we use the approximation

$$
g_{i j}=1-2 \phi
$$

This approximation is consistent with the linearized equation

$$
g_{\mu v}=\eta_{\mu v}+h_{\mu v}
$$

where $\eta_{\mu v}$ is the Minkowski metric with signature $(1,-1,-1,-1)$ and 


$$
h_{\mu v}=-2 \phi
$$

Limiting ourselves to 3 -space ( $\mathrm{d} t \equiv 0$ ) we obtain

$$
\mathrm{d} s^{2}=\frac{-1}{1+2 \phi}\left(\mathrm{d} x^{2}+\mathrm{d} y^{2}+\mathrm{d} z^{2}\right) .
$$

since the binomial expansion also yields

$$
\frac{1}{(1+\phi)^{2}}=\frac{1}{1+2 \phi}
$$

Equation (22) represents diagonals term $\mathrm{d} x_{i} \mathrm{~d} x_{i}$ with factor $h_{i i}$. In " $A$ primordial space-time metric" the representation of the gravitomagnetic field of the dense region of the field moving in the z-direction satisfies $h_{x y}-h_{y x}=0$. This angular momentum relation causes $\mathrm{d} x \mathrm{~d} y$ and $\mathrm{d} y \mathrm{~d} x$ terms to cancel, yielding Equation (22).

The moving density with $h_{x y} \neq 0, h_{y x}=-h_{x y}$ is seen to be compatible with Equation (22). The exact solution we've been considering however is based on the mass $M$ located at the origin. The spherical symmetry of this case implies $h_{x y}=h_{y x}=0 \quad$ (and cyclical iterations); also compatible with Equation (22). The alternative form of Equation (22) based on Equation (19) yields

$$
\mathrm{d} s^{2}=-(1-2 \phi)\left(\mathrm{d} x^{2}+\mathrm{d} y^{2}+\mathrm{d} z^{2}\right) .
$$

In order to touch base with general relativity, we use Equation (9) to observe that

$$
\phi=-\frac{g M}{r}
$$

Rewrite Equations (24) as

$$
\mathrm{d} s^{2}=-\left(1+\frac{2 g M}{r}\right)\left(\mathrm{d} x^{2}+\mathrm{d} y^{2}+\mathrm{d} z^{2}\right)
$$

Compare this to Ohanian and Ruffini's Equation (12) p.179 from the approximate metric tensor:

$$
g_{\mu \nu}=\left[\begin{array}{cccc}
1-2 g m / r & 0 & 0 & 0 \\
0 & -(1+2 g m / r) & 0 & 0 \\
0 & 0 & -(1+2 g m / r) & 0 \\
0 & 0 & 0 & -(1+2 g m / r)
\end{array}\right]
$$

Then compare to their space-time interval (Equation (13) p. 179):

$$
\begin{gathered}
\mathrm{d} s^{2}=g_{\mu \nu} \mathrm{d} x^{\mu} \mathrm{d} x^{\nu}=\left(1-\frac{2 g m}{r}\right) \mathrm{d} t^{2}-\left(1+\frac{2 g m}{r}\right)\left(\mathrm{d} x^{2}+\mathrm{d} y^{2}+\mathrm{d} z^{2}\right) \\
\mathrm{d} s^{2}=g_{\mu \nu} \mathrm{d} x^{\mu} \mathrm{d} x^{\nu}=(1+2 \phi) \mathrm{d} t^{2}-(1-2 \phi)\left(\mathrm{d} x^{2}+\mathrm{d} y^{2}+\mathrm{d} z^{2}\right)
\end{gathered}
$$

Let us use the alternative Minkowski metric $(-1,1,1,1)$ and let Newton's constant $g=1$ and speed of light $c=1$. In this case $\phi=-(M / r)$ and we write

$$
\mathrm{d} s^{2}=-\left(1+\frac{2 M}{r}\right)\left(\mathrm{d} x^{2}+\mathrm{d} y^{2}+\mathrm{d} z^{2}\right)+\left(1-\frac{2 M}{r}\right) \mathrm{d} t^{2}
$$




$$
\mathrm{d} s^{2}=-(1-2 \phi)\left(\mathrm{d} x^{2}+\mathrm{d} y^{2}+\mathrm{d} z^{2}\right)+(1+2 \phi) \mathrm{d} t^{2}
$$

This is exactly the form of Tolman's [22] expression (Equation (82.15)) for the Schwarzschild line element in approximate form (page 205). Similarly, Weinberg derives the Schwarzschild metric on page 180, Equation (8.2.12) using signature $(1,-1,-1,-1)$

$$
\begin{gathered}
\mathrm{d} \tau^{2}=\left[1-\frac{2 M g}{r}\right] \mathrm{d} t^{2}-\left[1-\frac{2 M g}{r}\right]^{-1} \mathrm{~d} r^{2}-\left[r^{2} \mathrm{~d} \theta^{2}+r^{2} \sin ^{2} \theta \mathrm{d} \phi^{2}\right] \\
\mathrm{d} \tau^{2}=[1+2 \phi] \mathrm{d} t^{2}-[1+2 \phi]^{-1} \mathrm{~d} r^{2}-\left[r^{2} \mathrm{~d} \theta^{2}+r^{2} \sin ^{2} \theta \mathrm{d} \phi^{2}\right]
\end{gathered}
$$

Many other general relativity books can be consulted for derivation of the Schwarzschild metric surrounding mass $M$ at the origin. Here we have used the encoding process to derive exactly the three-space metric representing the gravitational energy density equivalent information. For completeness and comparison I have included the time-time metric, $g_{00}$, but have not yet derived it; I do so in the next section. We conclude this section by observing that our approach of encoding energy density as geometry has yielded the Schwarzschild metric of curved space, the first exact solution to Einstein's equation. Rindler remarks that it is important to understand the spatial geometry of the Schwarzschild metric in order to apply to physical problems. Specifically:

"One way to visualizing the curved 3-space like that of the Schwarzschild lattice, whose metric is given by

$$
\mathrm{d} l^{2}=\frac{\mathrm{d} r^{2}}{1+2 \phi}+\left[r^{2}\left(\mathrm{~d} \theta^{2}+\sin ^{2} \theta \mathrm{d} \varphi^{2}\right)\right]
$$

is to pretend that it is really flat, but that it's rulers behave strangely..."

In Equation (31), we consider a radial ruler, [in which case $\mathrm{d} \theta=\mathrm{d} \varphi=0$ ] and note that the rulers shrink by factor $(1+2 \phi)^{1 / 2} \approx 1+\phi$. This is exactly the result for $\mathrm{d} x, \mathrm{~d} y$ and $\mathrm{d} z$ we achieved in Equation (14) by transforming from actual physical energy density in flat space (constant rulers) to the equivalent information encoded as geometry of curved space (variable metric). QED.

The computation necessary to derive the Schwarzschild metric in general relativity is far from beautiful; it is rather obtuse. Rindler, at the top of his preface quotes Bernhard Riemann

"The ideal is to reach proofs by comprehension rather than by computation."

By comprehending that we are encoding energy-density as geometry and drawing a few simple diagrams we have transformed from energy density in flat space (physical reality) to curved space with almost no computation and obtained the standard Schwarzschild solutions.

\section{The Time Metric Associated with Curved 3-Space}

Our focus in Section 2 was on "time dilation" in special relativity from the perspective of energy-time theory in absolute space and time. Sections 4 - 7 were focused on curved space and the associated "never solved" problem of gravitation- 
al energy in curved space. This led to the conclusion that gravitational energy density in flat space is banished by the equivalence principle, and yet the physical information associated with the density of the field cannot be simply discarded-it must be transformed into the geometry associated with variable metric coordinate systems. This conclusion, and associated perspective, led almost immediately to the appropriate metric for the non-trivial solution represented by the Schwarzschild metric. The assumption of absolute space and time underlying the physics of inertial clocks is based on defining "absolute time" as universal simultaneity, and hence our curved space solution holds at any time (slice) $t$ with $\mathrm{d} t \equiv 0$. This caused the time dimension to drop out of the Schwarzschild metric solution and reduce the problem to that of curved 3-space as derived in Section 8.

Recall that our goal was to encode the energy density in a unit cube in flat space $\mathrm{d} x \mathrm{~d} y \mathrm{~d} z=1$ in the variable metric $\mathrm{d} x^{\prime}, \mathrm{d} y^{\prime}, \mathrm{d} z^{\prime} \neq 1$ and we did so via Equation (14):

$$
\mathrm{d} x_{i}^{\prime}(\phi)=\frac{\mathrm{d} x_{i}}{1+\phi} .
$$

In other words we derived the variable metric $\mathrm{d} x_{i}^{\prime}$ from the flat space metric $\mathrm{d} x_{i}$ with its constant scale. This formulation is in Euclidean space and time derived by Equation (1). We now review the analysis context of Minkowski invariant defined by Equation (5)

$$
\mathrm{d} s^{2}=c^{2} \mathrm{~d} t^{2}-\mathrm{d} x^{2}-\mathrm{d} y^{2}-\mathrm{d} z^{2}=0
$$

It is called "invariant" because it does not vary with coordinate systems, hence

$$
\mathrm{d} s^{\prime 2}=c^{2} \mathrm{~d} t^{\prime 2}-\mathrm{d} x^{\prime 2}-\mathrm{d} y^{\prime 2}-\mathrm{d} z^{\prime 2}=0
$$

Let us rewrite this as

$$
\mathrm{d} s^{2}=0=\mathrm{d} t^{2}-\mathrm{d} r^{2}=\mathrm{d} t^{\prime 2}-\mathrm{d} r^{\prime 2}
$$

From our Equation (13) we express the coordinate differentials as $\mathrm{d} x_{i}$ and $\mathrm{d} x_{i}^{\prime}$ therefore we use

$$
\mathrm{d} s^{2}=g^{\mu v} \mathrm{~d} x_{\mu} \mathrm{d} x_{v}
$$

Hence, when I obtain (35) from Equation (33) I do so in the form

$$
\mathrm{d} t^{\prime 2}=\mathrm{d} r^{\prime 2}=\frac{\mathrm{d} r^{2}}{1+2 \phi}=g^{00} \mathrm{~d} x_{0} \mathrm{~d} x_{0}=g^{00} \mathrm{~d} t^{2}
$$

Minkowski-invariant-based energy-time theory derives from the equation of motion for photons

$$
\mathrm{d} r^{2}-c^{2} \mathrm{~d} t^{2}=0 \Rightarrow \frac{\mathrm{d} r^{2}}{\mathrm{~d} t^{2}}=c^{2} .
$$

Therefore, from Equation (35) we find

$$
\frac{\mathrm{d} r^{2}}{\mathrm{~d} t^{2}}=g^{00}(1+2 \phi)=c^{2} .
$$


The inverse metric $g_{00}=1 / g^{00}$ and when $c^{2}=1$ we obtain

$$
g^{00} g_{00}=1 \text { and } g^{00}(1+2 \phi)=1 \text {. }
$$

Therefore

$$
g_{00}=(1+2 \phi)
$$

is compared with Equations (29b) and (30b), and $g_{00}=(1+2 \phi)$ is found to be in exact agreement with those expressions of the Schwarzschild time-time metric. Therefore, our flat space distribution of field energy density, $\phi(\vec{r})$ encodes energy density information as geo(metric) information of curved space and the corresponding time (the conjugate of energy).

\section{Positional Dependence in the Local Field}

Energy-time analysis is based not on space-time symmetry, but on inertial mass $m=m_{0} \gamma(v)$; inertial factor $\gamma(v)$ relates to inertial mass, not to space and time. We extend this relation to general relativity [23] as

$$
m=m_{0} \gamma(\dot{\vec{r}}) \mathrm{e}^{\phi(\vec{r})}
$$

Here the mass of the particle depends upon the kinetic energy in a local absolute gravitational field as well as its position-dependent energy within a gravitational field with potential $\phi$. This inertial relation explains clock-slowing, as known and practiced in GPS.

Our "gravity free" definition of inertial mass, $m=\gamma m_{0}$ was found to include the kinetic energy in addition to rest mass: $m=m_{0}\left(1-v^{2} / c^{2}\right)^{-1 / 2}$. In a gravitational field the energy of a mass depends upon the Newtonian potential $\phi=-g M / r$ so let us naïvely add the gravitational term $2 \phi / c^{2}$ to the kinetic energy term as follows

$$
m=m_{0}\left[1-\left(\frac{v^{2}}{c^{2}}+\frac{2 \phi}{c^{2}}\right)\right]^{-1 / 2} \Rightarrow m_{0}\left[1+\frac{1}{2} \frac{v^{2}}{c^{2}}+\frac{\phi}{c^{2}}\right]
$$

yielding inertial mass and associated energies:

$$
\begin{gathered}
m c^{2}=m_{0} c^{2}+m_{0} v^{2} / 2+m_{0} \phi \\
/ \quad|\quad| \quad \mid \\
\text { total energy rest kinetic gravitational }
\end{gathered}
$$

This formulation demonstrates the dependence of inertial mass on velocity $\dot{\vec{r}}$ through the local gravitational field and on position $\vec{r}$ in the local gravitational field. Our simple addition of the gravitational potential $\phi=-g M / r$ is only intuitively justified, however Poisson and Will derive the same result in orthodox fashion based on the action

$$
S=-m_{0} c^{2} \int \mathrm{d} \tau=\int L \mathrm{~d} t
$$

where $\mathrm{d} \tau$ is the proper time after clock slowdown (speedup) is considered, and the Lagrangian 


$$
L=-m c \sqrt{-\eta_{\alpha \beta} \frac{\mathrm{d} r^{\alpha}}{\mathrm{d} t} \frac{\mathrm{d} r^{\beta}}{\mathrm{d} t}}
$$

is evaluated as

$$
L=-m c \sqrt{1-\frac{2 \phi}{c^{2}}-\frac{v^{2}}{c^{2}}}
$$

in which case

$$
L=-m_{0} c^{2}+m_{0} v^{2} / 2+m_{0} \phi
$$

As explained in Section 5, our energy-time Hamiltonian is completely compatible with the classical Lagrangian approach, therefore the Lagrangian in Equation (45) yields physics in agreement with Equation (42). We again see that the time-dependence of clocks follows from energy-based analysis rather than geometry.

Dimensional analysis shows that $\phi=g m / r$ has dimensions of velocity squared, $v^{2}$. Therefore to achieve the dimensionless factor we divide $\phi$ by $c^{2}=1$ and obtain $\frac{\phi}{c^{2}} \sim \frac{v^{2}}{c^{2}}$. Although we have obtained this via dimensional analysis, Weinberg (page 212) observes that it is a familiar result of Newtonian mechanics that the typical kinetic energy $M v^{2} / 2$ will be roughly of the same order of magnitude as the typical potential energy $g M^{2} / r$ hence

$$
M v^{2} / 2 \approx g M^{2} / r \Rightarrow v^{2} \sim \frac{g M}{r} .
$$

Moreover, a test particle in circular orbit of radius $r$ about a central mass $M$ will have velocity $v$ given by the exact formula $v_{g}^{2}=g M / r$.

In Equation (41), the special relativity term $\left[1+\frac{1}{2} \frac{v^{2}}{c^{2}}\right]$ represents positive energy, while $\phi=-g M / r$ is negative energy. Therefore

$$
m=m_{0}\left[1+\left[\frac{1}{2} \frac{v^{2}}{c^{2}}-\frac{v_{g}^{2}}{c^{2}}\right]\right]
$$

\section{Conclusions}

As noted, physicists have never solved the problem of including the energy-momentum of the gravitational field in Einstein's field equations. Based upon our analysis of this problem, we concur with Feynman, Weinberg, and others: the concept of curved space-time is not necessary for gravity. In contrast to this, we view gravity as a field whose energy density determines the physics in Euclidean 3-space, with absolute time spanning 3-space as universal simultaneity. Ontological arguments imply that both theories are not equally valid.

The equivalence principle is generally interpreted to imply that the field energy can be made to vanish locally despite universally agreed-upon rules that coordinate systems have no effect on physical reality. This "disappearance" of the gravitational energy of the field when curved coordinate frames appear has 
confused general relativists since Einstein proposed his geometric approach. In energy-time theory, the specification of field energy density in flat space determines the physics. If this information is removed, it must be replaced by some other source of information, and the curved coordinates supply this information. The actual gravitational field does not vanish; its energy density is normalized. That is, every corresponding differential volume in curved space coordinates, $\mathrm{d} x^{\prime} \mathrm{d} y^{\prime} \mathrm{d} z^{\prime}$, contains exactly one unit of energy. This approach is formulated herein for the primordial gravitational field and the field of mass $M$ at the origin. The normalization procedure determines the metric and that metric is exactly equal to the Schwarzschild solution. The energy of the gravitational field does not vanish in this approach; the normalization transfers the gravitational field information to the curved space coordinates.

One might wonder why physicists continue to work with "curved space-time" despite problems interpreting gravitational energy. Many physicists appear to believe in physical reality of curved space-time, as evidenced by a very recent paper in Phys Rev Letters [24]: “Traversable worm holes in Einstein-Dirac-Maxwell theory" constructs a specific example of a class of traversable worm holes in four space-time dimensions. The Heaviside equations, best suited for handling energy density, are generally believed to be useful only in "weak field" situations. However, Will and Poisson have remarked that this flat space approach is actually useful for very strong fields, but they offer no reason for this fact. In "The primordial principle of self-interaction", I derive the Heaviside equations with no "weak field" assumptions. This implies that the Heaviside equations work for all field strengths and thus present a complete theory of gravity. This is radically different from the prevailing view that only curved space-time equations are complete.

Dozens of theories of gravity exist; most are formulated in the $4 \mathrm{D}$ "curved space-time" of Riemann-Cartan. The current standard theory, ADM [25], splits $4 \mathrm{D} \rightarrow(3+1) \mathrm{D}$ and the space-time metric is replaced by the spatial-metric plus lapse and shift time steps. This $3+1$ split, compatible to a degree with absolute space and time, is required for the dynamical Hamiltonian formulation of ADM. Like ADM, Chen et al. base their theories on the Hamiltonian, which separates the equation into two sets (shown for electromagnetic field):

The initial value constraints: $\vec{\nabla} \cdot \vec{E}=\rho / \varepsilon_{0}, \vec{\nabla} \cdot \vec{B}=0$

The dynamic time equations: $\dot{\vec{B}}+\vec{\nabla} \times \vec{E}=0, \quad \vec{\nabla} \times \vec{B}=\mu_{0} \vec{J}+\dot{\vec{G}}$

The treatment of time is absolute, as in cosmological time-the universe-spanning dimension that establishes the NOW, missing from Einstein's theories. To this ADM-model, Chen et al. add a (Penrose) quasi-local 2D boundary. I interpret this as averaging over various-size cells, each of which contains a normalized unit-of-energy. Depending upon the 2D-quasi-local boundary chosen, this may help "undo" the normalization procedure and restore the variable energy-density upon which the curved-space formulation was based. The $3+$ 1)-split is invoked for Hamiltonian-based formulations which essentially require that the time derivative be singled out from the spatial derivatives, and thus 
cannot be truly four-covariant.

Why, if the non-solvable nature of Einstein-type theories of gravity as geometry is endemic, do physicists invariably believe the Einstein nonlinear field equations? I suspect that the key reason is their belief that linearized equations are appropriate only in the "weak field" approximation. In other words, no matter how preferable it might be to work gravity problems in flat space (instead of curved and flat space) the consensus belief is that the linear equations are incomplete. That is, curved space-time is physical reality. This belief is challenged by the recent development of the theory of gravity in The primordial principle of self-interaction, wherein the issue of "weak field" never arises; the development is good for all field strengths. Because the field interacts with its own mass, the nonlinear aspect of gravity appears in iterative dynamics. Feynman, Weinberg, and many others discuss the exact equivalence of the iterated linear equations to the nonlinear curved-space formalism, but the truth is effectively overridden by the "weak field" misnomer.

Physically, dynamical energy density physics can evolve over time, in contrast to the Schwarzschild metric, which is static and is solved for "all at once". The metric does not evolve to a solution; it is a priori. The implications of the primordial principle of self-interaction and of encoding energy-density in geometry are that it is actually the curved-space formalisms that are incomplete, and the gravitational field theory in Euclidean space represents physical reality. This ontological shift to a density-based formalism has significant consequences, some of which are being explored.

\section{Conflicts of Interest}

The author declares no conflicts of interest regarding the publication of this paper.

\section{References}

[1] Chen, C.-N., et al. (2015) International Journal of Modern Physics D, 24, Article ID: 1530026 .

[2] Nozick, R. (2001) Invariance: The Structure of the Objective World. Belknap-Harvard, Cambridge, MA.

[3] Hestenes, D. (1986) New Foundations for Classical Mechanics. 2nd Edition, Kluwer Academic Publishers, Dordrecht. https://doi.org/10.1007/978-94-009-4802-0

[4] Klingman, E. (2020) Journal of Modern Physics, 11, 1950-1968. https://doi.org/10.4236/jmp.2020.1112123

[5] Lucas and Hodgson (1990) Space-Time and Electromagnetics. Oxford University Press, Oxford.

[6] Rindler, W. (1991) Introduction to Special Relativity. 2nd Edition, Oxford Science Pub., Oxford.

[7] Cannoni, M. (2016) Lorentz Invariant Relative Velocity and Relativistic Binary Collisions.

[8] Klingman, E. (2018) Everything's Relative, Or Is It. 
[9] Fromholz, P., Will, C. and Poisson, E. (2013) The Schwarzschild Metric: It's the Coordinates, Stupid.

[10] Weinberg, S. (1972) Gravitation and Cosmology. John Wiley \& Sons, New York.

[11] Poisson, E. and Will, C. (2014) Gravity: Newtonian, Post-Newtonian, Relativistic. Cambridge University Press, Cambridge.

[12] Misner, C.W., Thorne, K.S. and Wheeler, J.A. (1973) Gravitation. W.H. Freeman and Company, New York.

[13] Feynman, R. (1995) Feynman Lectures on Gravitation. Westview Press, Boulder.

[14] Vishwakarma, R. (2013) Gravity of $R \mu v=0:$ A New Paradigm in GR.

[15] Klingman, E. (2019) Prespacetime Journal, 10, 671-680.

[16] Heaviside, O. (1893) The Electrician, 31, 281-282.

[17] Einstein, A. (1952) Relativity: The Special and General Theory. Crown Publishers Inc., New York.

[18] Ohanian, H. and Ruffini, R. (2013) Gravitation and Spacetime. 3rd Edition, Cambridge University Press, Cambridge. https://doi.org/10.1017/CBO9781139003391

[19] Verlinde, E. (2010) On the Origin of Gravity and the Laws of Newton.

[20] Klingman, E. (2020) Journal of Modern Physics, 12, 65-81. https://doi.org/10.4236/jmp.2021.122007

[21] Yilmaz, H. (1992) Il Nuovo Cimento, 107B, 941-960. https://doi.org/10.1007/BF02899296

[22] Tolman, R. (1962) Relativity, Thermodynamics and Cosmology. University Press, Oxford.

[23] Robertson, S. (2019) Prespacetime Journal, 10, 69-74.

[24] Blazques-Salcedo, J. (2021) Physical Review Letters, 126, Article ID: 101102. https://doi.org/10.1103/PhysRevLett.126.101102

[25] Arnowitt, R., Deser, S. and Misner, C. (2004) The Dynamics of General Relativity. 